Landslide-water Interaction for Partially Submerged Landslide

\title{
Landslide-water Interaction for Partially Submerged Landslide
}

\author{
Jeevan Kafle ${ }^{1,2}$, Bhadra Man Tuladhar ${ }^{1}$ \\ ${ }^{1}$ School of Science, Kathmandu University, Dhulikhel, Kavre, Nepal. \\ 2 Central Department of Mathematics, Tribhuvan University, Kathmandu, Nepal. \\ Correspondence to: Jeevan Kafle, email: jkafle@student.ku.edu.np
}

\begin{abstract}
Tsunamis are long water waves triggered by impulsive geologic events. Tsunamis generated by landslides may be classified based on the initial position of the landslide as subaerial, partially submerged or submarine landslide generated tsunamis depending on the initial position of the landslide relative to the water depth. Here we present and discuss a simulation related to a partially submerged landslide in a quiescent reservoir by using the general two-phase mass flow model (Pudasaini, 2012) to observe the explicit evolution and propagation of surface tsunami waves, and solid waves as submarine mass movement. Tsunami waves are reflected as they impact the right coast and the lateral walls of the reservoir. The study of wave propagation, reflection and interaction as well as the submarine mass movement enhance our understanding on the tsunami-related phenomena and the turbidity current in water bodies and coastal areas.
\end{abstract}

Keywords: Debris flows, Two-phase mass flows, Landslide-water interaction, Partially submerged landslides, High-resolution methods.

\section{Introduction}

Submarine and subaerial landslides, debris flows and debris avalanches may generate super tsunami waves as they are triggered and impact geometrically confined water bodies (reservoirs, lakes, fjords, and rivers) causing potentially damage of population and infrastructures in coastal emplacements due to extreme tsunami run-up [4, 12, 21]. The initial position of the landslide initiation relative to the water surface categorizes the landslide-water interaction into subaerial, partially submerged, or submarine landslides [5, 8, 16. A partially submerged landslide may generate tsunami waves in water bodies by a rapid or an impulsive transfer of momentum from the landslide mass to the water body, and the surface displaces water away from the impact region during the impact, and subaqueous runout [15]. The tsunami wave heights tend to decrease with increasing submergence of the initial position of the landslide [12. The process of partially submerged landslide generated tsunamis can be divided into the following categories: the mechanism of tsunamigenic landslides, the tsunami wave generation in the near field region, the tsunami wave propagation and the hazards associated with tsunami wave runup and impact [9, 12, 15]. Tsunami wave shoaling, diffraction, refraction and wave breaking depend upon the initial volume of the landslide mass, bathymetry and the topography as well as the vulnerability of the coastal region [15]. For planning any submerged landslide generated tsunami control scheme, assessment of the hazard becomes necessary, and the estimation of the dynamic parameters, like velocity, impact forces and kinetic energy are major inputs for designing the control measures [1, 2. The tsunami prevention system consists of forecasting, warning, evacuation, public education, drills, inheritance of disaster culture, and the relief operation after disaster 22. Although there were substantial attempts made in the past, all these events and their associated dynamics, and impacts are yet to be satisfactorily investigated further. The development and applications of the mitigation measures need further improvements. In order to mitigate mountain and coastal hazards and integrity of hydraulic power plants, it is very essential to properly investigate partially submerged landslide and tsunami [11, 14].

Along with buoyancy, two-phase mass flow, Pudasaini [17] developed a generalized two-phase mass flow model unifying existing avalanche and debris flow theories. The model includes buoyancy, and also three other dominant physical aspects of the flow: enhanced non-Newtonian viscous stress, virtual mass and generalized drag [17. In this model, the solid phase stress is described by Mohr-Coulomb plasticity, while the fluid stress is modelled as a non-Newtonian viscous stress that is enhanced by the solid-volumefraction gradient. A generalized interfacial momentum transfer includes buoyancy, virtual mass forces, and a generalized drag force to cover both solid-like and fluid-like drags. Simulations of real two-phase debris mass flows, both in subaerial and submarine environment, were carried out by Pudasaini [17, 18, for the 
first time for the explicit evolution of the solid and fluid phases as the debris mass collapses and slides down the slope. The simulations were carried on geometrically two-dimensional setting and for two-phase flows impinging a still reservoir to produce tsunamis upon impact that generated submarine debris slides along the reservoir bed. For an idealized geometry, Kafle [7] and Kafle et al. 8] simulated two-phase and threedimensional subaerial flows impacting a fluid reservoir, the splash, tsunami generation, and submarine debris mass movements. Mergili et al. [13] studied the complex hydro-geomorphic process chains in a multi-lake outburst flood in the Santa Cruz Valley in Cordillera Blanca, Peru. Kafle et al. [9] present first-ever three-dimensional, high-resolution simulation results for a two-phase debris mass impacting a fluid reservoir installed with obstacles of different shapes, sizes, numbers, and at different subaerial slopes and bathymetric positions.

The present work considers the general mass flow model by Pudasaini [17, and mainly focuses on the numerical modeling of the impulse waves generated by the partially submerged landslides along with the submarine mass movement. Generally, partially submerged landslides occur due to the shore instability created by the water-shore interactions. This is a reason for choosing partially submerged landslide.

\section{Governing Equations}

In the general two-phase mass flow model (Pudasaini, 2012) [17, the subscripts $s$ and $f$ stand for the solid and the fluid phases respectively. The mass and momentum balances for solid and fluid in the down-slope $(x)$ and the cross-slope $(y)$ directions are given by

$$
\begin{aligned}
& \frac{\partial}{\partial t}\left(\alpha_{s} h\right)+\frac{\partial}{\partial x}\left(\alpha_{s} h u_{s}\right)+\frac{\partial}{\partial y}\left(\alpha_{s} h v_{s}\right)=0, \\
& \frac{\partial}{\partial t}\left(\alpha_{f} h\right)+\frac{\partial}{\partial x}\left(\alpha_{f} h u_{f}\right)+\frac{\partial}{\partial y}\left(\alpha_{f} h v_{f}\right)=0 \\
& \frac{\partial}{\partial t}\left[\alpha_{s} h\left(u_{s}-\gamma \mathcal{C}\left(u_{f}-u_{s}\right)\right)\right]+\frac{\partial}{\partial x}\left[\alpha_{s} h\left(u_{s}^{2}-\gamma \mathcal{C}\left(u_{f}^{2}-u_{s}^{2}\right)+\frac{\beta_{x_{s}} h}{2}\right)\right] \\
& +\frac{\partial}{\partial y}\left[\alpha_{s} h\left(u_{s} v_{s}-\gamma \mathcal{C}\left(u_{f} v_{f}-u_{s} v_{s}\right)\right)\right]=h S_{x_{s}}, \\
& \frac{\partial}{\partial t}\left[\alpha_{s} h\left(v_{s}-\gamma \mathcal{C}\left(v_{f}-v_{s}\right)\right)\right]+\frac{\partial}{\partial x}\left[\alpha_{s} h\left(u_{s} v_{s}-\gamma \mathcal{C}\left(u_{f} v_{f}-u_{s} v_{s}\right)\right)\right] \\
& +\frac{\partial}{\partial y}\left[\alpha_{s} h\left(v_{s}^{2}-\gamma \mathcal{C}\left(v_{f}^{2}-v_{s}^{2}\right)+\frac{\beta_{y_{s}} h}{2}\right)\right]=h S_{y_{s}} \\
& \frac{\partial}{\partial t}\left[\alpha_{f} h\left(u_{f}+\frac{\alpha_{s}}{\alpha_{f}} \mathcal{C}\left(u_{f}-u_{s}\right)\right)\right]+\frac{\partial}{\partial x}\left[\alpha_{f} h\left(u_{f}^{2}+\frac{\alpha_{s}}{\alpha_{f}} \mathcal{C}\left(u_{f}^{2}-u_{s}^{2}\right)+\frac{\beta_{x_{f}} h}{2}\right)\right] \\
& +\frac{\partial}{\partial y}\left[\alpha_{f} h\left(u_{f} v_{f}+\frac{\alpha_{s}}{\alpha_{f}} \mathcal{C}\left(u_{f} v_{f}-u_{s} v_{s}\right)\right)\right]=h S_{x_{f}} \\
& \frac{\partial}{\partial t}\left[\alpha_{f} h\left(v_{f}+\frac{\alpha_{s}}{\alpha_{f}} \mathcal{C}\left(v_{f}-v_{s}\right)\right)\right]+\frac{\partial}{\partial x}\left[\alpha_{f} h\left(u_{f} v_{f}+\frac{\alpha_{s}}{\alpha_{f}} \mathcal{C}\left(u_{f} v_{f}-u_{s} v_{s}\right)\right)\right] \\
& +\frac{\partial}{\partial y}\left[\alpha_{f} h\left(v_{f}^{2}+\frac{\alpha_{s}}{\alpha_{f}} \mathcal{C}\left(v_{f}^{2}-v_{s}^{2}\right)+\frac{\beta_{y_{f}} h}{2}\right)\right]=h S_{y_{f}} .
\end{aligned}
$$


in which

$$
\beta_{x_{s}}=\varepsilon K_{x} p_{b_{s}}, \beta_{y_{s}}=\varepsilon K_{y} p_{b_{s}}, \beta_{x_{f}}=\beta_{y_{f}}=\varepsilon p_{b_{f}}, p_{b_{f}}=-g^{z}, p_{b_{s}}=(1-\gamma) p_{b_{f}} .
$$

Here, $p_{b_{f}}$ and $p_{b_{s}}$ are the effective fluid and solid pressures at the base. The equations (1)-(2) are depthaveraged mass balance equations for solid and fluid phases respectively. The last four equations are depthaveraged momentum balances for solid (3)-(4) and fluid (5)-(6) in the $x$ - and $y$-directions respectively. The source terms are (Pudasaini, 2012):

$$
\begin{aligned}
& S_{x_{s}}=\alpha_{s}\left[g^{x}-p_{b_{s}}\left(\frac{u_{s}}{\left|\mathbf{u}_{\mathbf{s}}\right|} \tan \delta+\varepsilon \frac{\partial b}{\partial x}\right)\right]-\varepsilon \alpha_{s} \gamma p_{b_{f}}\left[\frac{\partial h}{\partial x}+\frac{\partial b}{\partial x}\right] \\
& +C_{D G}\left(u_{f}-u_{s}\right)\left|\mathbf{u}_{f}-\mathbf{u}_{s}\right|^{J-1}, \\
& S_{y_{s}}=\alpha_{s}\left[g^{y}-p_{b_{s}}\left(\frac{v_{s}}{\left|\mathbf{u}_{\mathbf{s}}\right|} \tan \delta+\varepsilon \frac{\partial b}{\partial y}\right)\right]-\varepsilon \alpha_{s} \gamma p_{b_{f}}\left[\frac{\partial h}{\partial y}+\frac{\partial b}{\partial y}\right] \\
& +C_{D G}\left(v_{f}-v_{s}\right)\left|\mathbf{u}_{f}-\mathbf{u}_{s}\right|^{J-1}, \\
& S_{x_{f}}=\alpha_{f}\left[g^{x}-\varepsilon\left[\frac{1}{2} p_{b_{f}} \frac{h}{\alpha_{f}} \frac{\partial \alpha_{s}}{\partial x}+p_{b_{f}} \frac{\partial b}{\partial x}-\frac{1}{\alpha_{f} N_{R}}\left\{2 \frac{\partial^{2} u_{f}}{\partial x^{2}}+\frac{\partial^{2} v_{f}}{\partial y \partial x}+\frac{\partial^{2} u_{f}}{\partial y^{2}}-\frac{\chi u_{f}}{\varepsilon^{2} h^{2}}\right\}\right.\right. \\
& +\frac{1}{\alpha_{f} N_{R_{\mathcal{A}}}}\left\{2 \frac{\partial}{\partial x}\left(\frac{\partial \alpha_{s}}{\partial x}\left(u_{f}-u_{s}\right)\right)+\frac{\partial}{\partial y}\left(\frac{\partial \alpha_{s}}{\partial x}\left(v_{f}-v_{s}\right)+\frac{\partial \alpha_{s}}{\partial y}\left(u_{f}-u_{s}\right)\right)\right\} \\
& \left.\left.-\frac{\xi \alpha_{s}\left(u_{f}-u_{s}\right)}{\varepsilon^{2} \alpha_{f} N_{R_{\mathcal{A}}} h^{2}}\right]\right]-\frac{1}{\gamma} C_{D G}\left(u_{f}-u_{s}\right)\left|\mathbf{u}_{f}-\mathbf{u}_{s}\right|^{J-1} \\
& S_{y_{f}}=\alpha_{f}\left[g^{y}-\varepsilon\left[\frac{1}{2} p_{b_{f}} \frac{h}{\alpha_{f}} \frac{\partial \alpha_{s}}{\partial y}+p_{b_{f}} \frac{\partial b}{\partial y}-\frac{1}{\alpha_{f} N_{R}}\left\{2 \frac{\partial^{2} v_{f}}{\partial y^{2}}+\frac{\partial^{2} u_{f}}{\partial x \partial y}+\frac{\partial^{2} v_{f}}{\partial x^{2}}-\frac{\chi v_{f}}{\varepsilon^{2} h^{2}}\right\}\right.\right. \\
& +\frac{1}{\alpha_{f} N_{R_{\mathcal{A}}}}\left\{2 \frac{\partial}{\partial y}\left(\frac{\partial \alpha_{s}}{\partial y}\left(v_{f}-v_{s}\right)\right)+\frac{\partial}{\partial x}\left(\frac{\partial \alpha_{s}}{\partial y}\left(u_{f}-u_{s}\right)+\frac{\partial \alpha_{s}}{\partial x}\left(v_{f}-v_{s}\right)\right)\right\} \\
& \left.\left.-\frac{\xi \alpha_{s}\left(v_{f}-v_{s}\right)}{\varepsilon^{2} \alpha_{f} N_{R_{\mathcal{A}}} h^{2}}\right]\right]-\frac{1}{\gamma} C_{D G}\left(v_{f}-v_{s}\right)\left|\mathbf{u}_{f}-\mathbf{u}_{s}\right|^{J-1},
\end{aligned}
$$

where,

$$
\begin{aligned}
& C_{D G}=\frac{\alpha_{s} \alpha_{f}(1-\gamma)}{\left[\varepsilon \mathcal{U}_{T}\left\{\mathcal{P} \mathcal{F}\left(R e_{p}\right)+(1-\mathcal{P}) \mathcal{G}\left(R e_{p}\right)\right\}\right]^{J}}, \gamma=\frac{\rho_{f}}{\rho_{s}}, \mathcal{F}=\frac{\gamma}{180}\left(\frac{\alpha_{f}}{\alpha_{s}}\right)^{3} R e_{p}, \\
& \mathcal{G}=\alpha_{f}^{M\left(R e_{p}\right)-1}, R e_{p}=\frac{\rho_{f} d \mathcal{U}_{T}}{\eta_{f}}, N_{R}=\frac{\sqrt{g L} H \rho_{f}}{\alpha_{f} \eta_{f}}, N_{R_{\mathcal{A}}}=\frac{\sqrt{g L} H \rho_{f}}{\mathcal{A} \eta_{f}}
\end{aligned}
$$

In the above equations, $x, y$ and $z$ are coordinates along the down-slope, cross-slope and the normal directions, respectively; and $g^{x}, g^{y}$ and $g^{z}$ are the respective components of gravitational acceleration. $L$ and $H$ are the typical length and depth of the flow, $\varepsilon=H / L$ is the aspect ratio, and $\mu=\tan \delta$ is the basal friction coefficient. $C_{D G}$ is the generalized drag coefficient. The generalized drag is modeled by a linear combination of $\mathcal{F}$ and $\mathcal{G}$. $J=1$ or 2 respectively, are associated with the simple linear (laminar-type, at low velocity) or quadratic (turbulent-type, at high velocity) drag. $\mathcal{U}_{T}$ is the terminal velocity of a particle and $\mathcal{P} \in[0,1]$ is an interpolation parameter that combines the solid-like $(\mathcal{G})$ and fluid-like $(\mathcal{F})$ drag contributions to flow resistance. $\mathcal{P}=0$ is more suitable when solid particles are moving through a fluid. On other hand, $\mathcal{P}=1$ is more suitable for flows of fluids through dense packing of grains (Pudasaini, 2012). $\gamma$ is the density ratio, $\mathcal{C}$ is the virtual mass coefficient (kinetic energy of the fluid phase induced by solid particles), 
Journal of Nepal Mathematical Society (JNMS), Vol. 1, Issue 1 (2018); J. Kafle, B. M. Tuladhar

$\eta_{f}$ is the fluid viscosity, $M$ is a function of the particle Reynolds number $\left(R_{e_{p}}\right), \chi$ includes vertical shearing of fluid velocity, and $\xi$ takes into account different distributions of $\alpha_{s}$. $\mathcal{A}$ is the mobility of the fluid at the interface, and $N_{R}$ and $N_{R_{\mathcal{A}}}$ respectively, are the quasi-Reynolds number and mobility-Reynolds number associated with the classical Newtonian and enhanced non-Newtonian fluid viscous stresses. Slope topography is given by $b=b(x, y)$. Initial condition is describe by the initial geometry (slope surface and water reservoir), the rectangular initial debris mass and the parameter values chosen for simulation. We have the kinematic free-surface and bottom boundary conditions: the top surface is traction-free and Coulomb sliding (for solid), and no-slip (for fluid) conditions are satisfied at the flow base.

The model equations (1)-(6) are written as well-structured, non-linear hyperbolic-parabolic partial differential equations in conservative form [17. These model equations are used to compute the debris depth $h$, solid volume fraction $\alpha_{s}$, velocity components for solid $\left(u_{s}, v_{s}\right)$, and for fluid $\left(u_{f}, v_{f}\right)$ in $x$ - and $y$-directions, respectively, as functions of space and time (Fig. 11). The model equations are solved in conservative variables $\mathbf{W}=\left(h_{s}, h_{f}, m_{s_{x}}, m_{f_{x}}, m_{s_{y}}, m_{f_{y}}\right)^{t}$, where $h_{s}=\alpha_{s} h, h_{f}=\alpha_{f} h$ are the solid and fluid contributions to the debris, or the flow height; and $m_{s_{x}}=\alpha_{s} h u_{s}, m_{f_{x}}=\alpha_{f} h u_{f} ; m_{s_{y}}=\alpha_{s} h v_{s}, m_{f_{y}}=\alpha_{f} h v_{f}$ are respectively the solid and fluid momenta in $x$ - and $y$-directions. This facilitates numerical integration even when shocks are formed in the field variables [17. The high-resolution shock-capturing Total Variation Diminishing Non-Oscillatory Central (TVD-NOC) scheme [3, 8, 10, 13, 18, 19, 20] has been employed.

\section{Simulation Results and Discussion}

For our simulation purpose, we consider a (geometrically) three-dimensional and two-phase partially submerged debris flow that hits a fluid reservoir. The upper part of the channel is inclined downwards $\left(\zeta_{0}=45^{\circ}\right)$ to the right and is flat in the lateral direction (Fig. 1). The left end of the reservoir meets an inclined surface (plane) at $x=170 \mathrm{~m}$, where the initial water height is zero. The inclined surface continues to $x=200 \mathrm{~m}$, where the water height is $h=30 \mathrm{~m}$ to the right of $x=200 \mathrm{~m}$, the bed of the reservoir is horizontal and the initial fluid height remains constant, i.e., $h=30 \mathrm{~m}$ from $x=200 \mathrm{~m}$ to $x=300 \mathrm{~m}$. Initially at $t=0 \mathrm{~s}$, the debris is in the cuboidal form $(50 \mathrm{~m} \leq x \leq 190 \mathrm{~m} ;-25 \mathrm{~m} \leq y \leq 25 \mathrm{~m})$ (Fig. 1) of constant total debris height $40 \mathrm{~m}$. The initial debris mass in the upper part of the slope is uniformly distributed as a homogeneous mixture of $70 \%$ solid $\left(\alpha_{s}=0.7\right)$ and $30 \%$ fluid $\left(\alpha_{f}=0.3\right)$. Similarly, initially the quiescent reservoir consists $2 \%$ solid and $98 \%$ fluid. The other parameter values chosen for simulation are: $\phi=45^{\circ}, \delta=15^{\circ}, \rho_{f}=1,100 \mathrm{~kg} \mathrm{~m}^{-3}, \rho_{s}=2,900 \mathrm{~kg} \mathrm{~m}^{-3}, N_{R}=30,000, N_{R_{\mathcal{A}}}=1,000, R e_{p}=1$, $\mathcal{U}_{T}=5.0 \mathrm{~m} \mathrm{~s}^{-1}, \mathcal{P}=0.75, J=1, \chi=3, \xi=5, \mathcal{C}=0.5$.

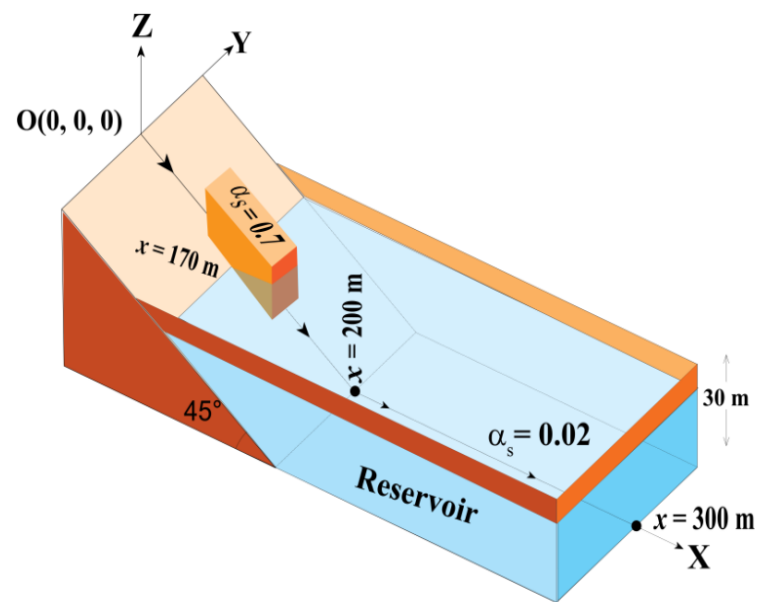

Figure 1: A sketch of the initial configuration of a debris mass on an inclined slab and a fluid reservoir in the downstream. Initially, the left end of the reservoir has the water height zero, which, due to the slope, gradually increases to its maximum of height of $30 \mathrm{~m}$ from the down-slope coordinate $x=170 \mathrm{~m}$ to $x=200$ $\mathrm{m}$. Beyond this, from $x=200 \mathrm{~m}$ to $x=300 \mathrm{~m}$, the initial fluid is at a constant height of $30 \mathrm{~m}$. 
Simulation results for the evolution of the submerged debris flows, and the tsunami generation by the debris impact and the subsequent complex fluid waves have been presented here. Figure 2 presents some basic results in terms of the partially submerged total debris depth (the sum of the solid and the fluid heights) along with the evolution of the solid phase, the penetration of the debris into the reservoir, the motion of the submarine debris flows, and the complex wave interactions. For the sake of simplicity, the simulation results are plotted (Fig. 22 with a bird's eye perspective. Nevertheless, the colour map given alongside in each subplot clearly provides necessary information about the flow depth and the mass distribution. Here, for the clear visualization, the colour patches are generated by filling the gaps between the contours. From $t=0 \mathrm{~s}$ (left panel a), the debris mass has just started to deform and move on the inclined plane, mainly in the down-slope direction, but also in the cross-slope direction. A bit of debris mass also moves upslope due to pressure gradient. The pressure gradient causes the dispersion in all direction. However, due to gravity, the mass is sheared mainly in downslope direction. As the mass is continuously spreading on the inclined plane, debris height has been substantially decreased.

The dynamics is more smooth and water wave is clearly visible even at $t=1 \mathrm{~s}$. Nevertheless, as the mass just accelerates downslope the motion is mainly pressure-driven and there is not much of inertia in the inception of the motion so that the tsunami with large extents could not be created in the early stages of time. The special evolution of the total debris is seen at $t=1 \mathrm{~s}$ when the debris mass is triggered due to gravity and pressure, it shears both in downslope and cross-slope direction. As initially the debris mass is superposed in the reservoir, mainly the initial fluid height, and also a bit of the solid material height is added to the initial debris that effectively increases the front of the initial material making it negatively sloped. This then results in some backward motion of the mass in the early stage of the motion. This is the reason for the tail moving a bit up slope for $t \leq 3 \mathrm{~s}$. The frontal part of the debris mass has created a tsunami in its vicinity. As time progresses, the tsunami propagates both down slope and cross slope with the increased amplitude (nearly $15 \mathrm{~m}$ at $t=3 \mathrm{~s}$ ). At $t=3 \mathrm{~s}$ and $t=5 \mathrm{~s}$, the blue tails represent the rear part of the debris mass. At $t=3 \mathrm{~s}$, the tail goes back due to the impact of the debris with the water surface. At $t=5 \mathrm{~s}$, a small three-dimensional hydrodynamic impact vacuum [18, or crater [6], which increases and retains for some more time. From $t=3 \mathrm{~s}$ to $t=5 \mathrm{~s}$, the tsunami propagates more rapidly in both down slope and cross-slope direction, with a little decrease in tsunami intensity.

As the debris mass pushes the reservoir fluid and accelerates downslope high amplitude and stronglycurved tsunami waves are observed along the left shore-line, creating a big trough in the center of the reservoir-shore. The debris mass almost penetrates the reservoir and submerges fully after at $t=7 \mathrm{~s}$. Then, due to the ceasing of the debris mass flow, the left coast tsunami begins propagating a bit upslope to fill the previously generated debris impact vacuum $[18$. At $t=7 \mathrm{~s}$, the tsunami wave front has already impacted the right coast as well as the lateral walls of the reservoir. The tsunami amplitude at the central right coast increases nearly to $20 \mathrm{~m}$. The increase in tsunami amplitude is due to the reflection, interaction and interference. The changing geometry of tsunami waves from $t=7 \mathrm{~s}$ to $t=9 \mathrm{~s}$ clearly shows that the tsunami waves at the lateral walls further propagate downslope and those in right coast propagates cross slope. This results in the localized high intensity tsunami waves at the two right corners of the reservoir pointing diagonally.

The total and the fluid-only geometric evolutions are qualitatively similar and so are not presented here. This is so due to the dominant amount of the fluid in the reservoir [8]. However, the dynamics of the solid phase is completely different, mainly due to a submarine mass flow. The flow behaviour of the solid-only part has been presented in Fig. $2 \mathrm{~B}$ as extracted from the total debris from Fig. $2 \mathrm{~A}$. As the mass is released, the front and backward rarefied and dispersed in all direction but advects more down slope and accelerating mainly due to the gravity. This is because the initial mass is in the form of a cuboid. Due to the slope, solid mass accelerates rapidly and a solid maximum formed at the transition between the slope and the horizontal surface. As the deposition morphology has already been started at this time slice, the solid depth increases to $30 \mathrm{~m}$. At $t=5 \mathrm{~s}$, the main solid body, its head and tail all move further downslope. The solid maximum is further dispersed, and so the solid depth decreases to $22 \mathrm{~m}$. The solid mass is completely submerged into the reservoir already at $t=7 \mathrm{~s}$. At $t=9 \mathrm{~s}$, the solid tail further pushes the main solid body. The two lateral ankers like shapes in $t=3 \mathrm{~s}$ and $t=5 \mathrm{~s}$ are caused by the splash of the debris mass into water, created mainly by the frontal debris head and the main debris body. From $t=7 \mathrm{~s}$ onward, these structures slowly diffuse.

Due to relatively slow debris motion, the drag is less and that the dilute front could thus move sub- 
Journal of Nepal Mathematical Society (JNMS), Vol. 1, Issue 1 (2018); J. Kafle, B. M. Tuladhar

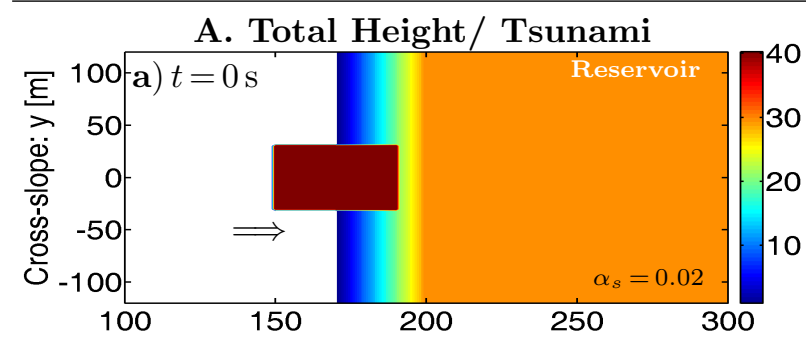

B. Solid Height/ Submarine Flows
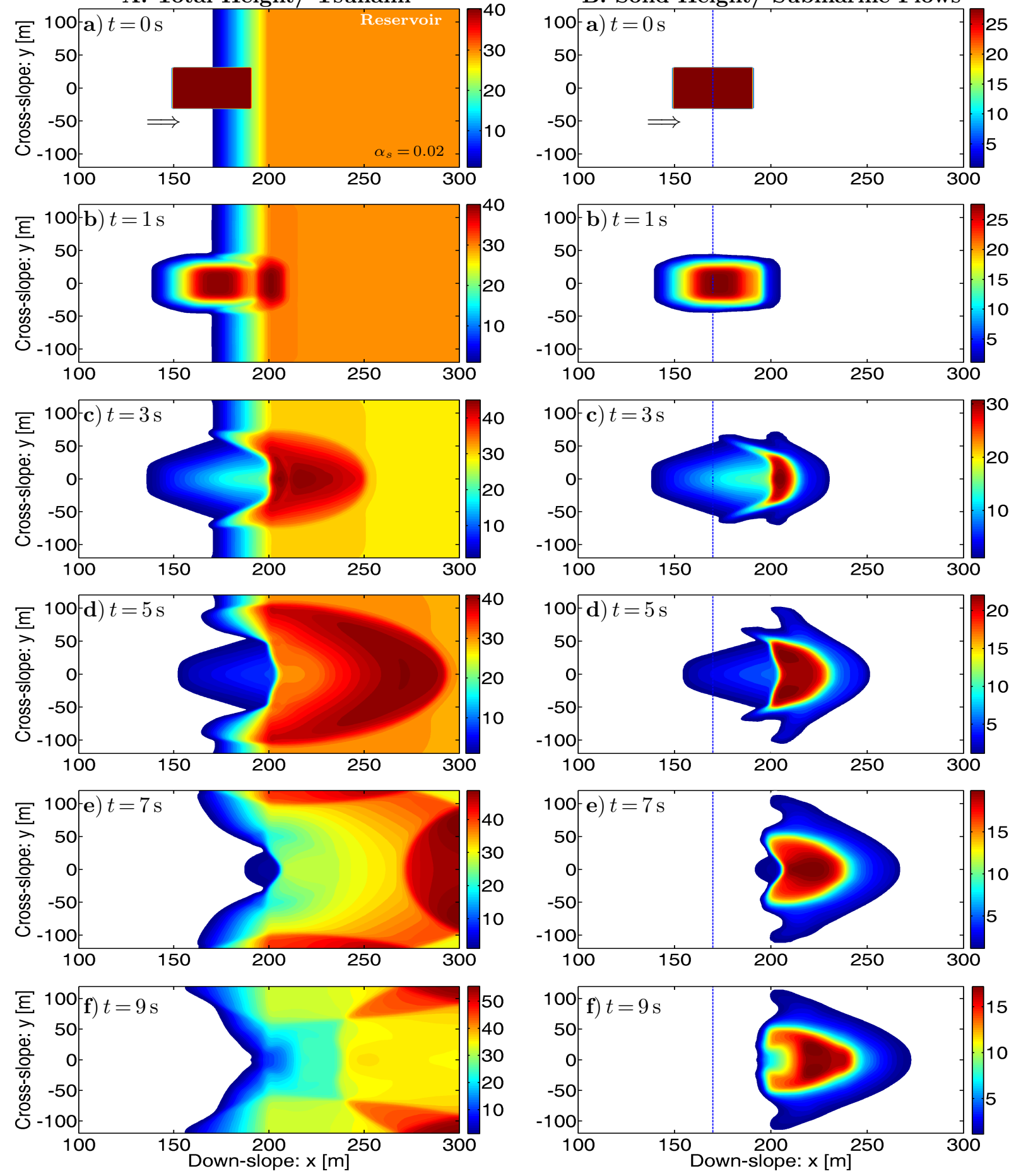

Figure 2: A: Total height of the debris mass and tsunami waves when initially the reservoir contains $2 \%$ solid grains and 98\% fluid, while the initial partially submerged debris consists of $70 \%$ solid grains and $30 \%$ fluid. As time elapses, the formation, amplification, propagation, reflection and intersection of coupled three-dimensional tsunami waves are observed as partially submerged two-phase debris mass further plunges into the quiescent water reservoir. B: Evolution of the solid phase only or submarine landslide. As soon as mass is released, solid mass disperses in all directions, mainly in downslope direction. After $t=3 \mathrm{~s}$, the solid mass forms a very special forward propagating laterally wide and curved solid-wave. The vertical line at $x=170 \mathrm{~m}$ indicates the left coast of the reservoir. The arrows in the top panels indicate the flow direction. 
Landslide-water Interaction for Partially Submerged Landslide

stantially farther. The solid shows more and immediate depositional behavior, and the major portion of the solid material begins to deposit just before the flat base, i.e., in the inclined submerged portion of the reservoir. Overall, the depositional characteristics is more smooth, but the main deposition reveals a backward propagating shock wave from $t=1 \mathrm{~s}$ to $t=3 \mathrm{~s}$. After that, the shock diffuses a bit. Furthermore, the major portion of the solid mass fraction is elongated in the cross-slope whereas the dispersed and dilute mass is elongated in the down-slope direction. These are fundamentally different characteristics observed here when initial debris mass is partially submerged.

The submarine mass movement as shown in $t=7 \mathrm{~s}$ and $t=9 \mathrm{~s}$ (Fig. 2B) takes a parabolic form as the solid mass advects in down-slope direction as well as disperses along cross slope direction. The special shape is due to the fact that the solid at front looses momentum in the run out zone where as the rear part is still advecting downslope and merges into the main debris body. Due to the interaction of the solid mass with the fluid reservoir, from $t=3 \mathrm{~s}$ (right panel c) to $t=9 \mathrm{~s}$ (right panel f), it forms a very special forward propagating laterally wide and curved solid-wave. Figure $2 \mathrm{~B}$ (right) also shows the propagation of the turbidity current and diffusion of the solid waves in the front and the lateral margins. These novel observations help us for the proper understanding of mixing and separation between phases, generation and propagation of special solid and fluid structures, and transitions of submarine landslide and debris induced tsunamis in mountain lakes, channels, and reservoirs, the associated dynamics of turbidity current and sediment transport by submarine landslides. These results may be extended and applied to hazard mitigation, prevention and solving relevant engineering or environmental task.

\section{Conclusion}

Here, we considered the general mass flow model by Pudasaini (2012) 17] to mainly focus on the numerical modeling of the impulse waves generated by the partially submerged landslides, along with the submarine mass flows by employing a high-resolution shock-capturing numerical scheme. The formation, amplification, propagation, reflection and intersection of coupled three-dimensional tsunami waves are observed as a partially submerged two-phase debris mass impacts the quiescent water reservoir. The simulation shows that the tsunami waves advect both longitudinally and laterally. This results in the localized high intensity tsunami waves at the two right corners of the reservoir pointing diagonally. During the collapse of the debris mass, the tail moves a bit backward due to negative slope of its initial setting. At early time stages, the two lateral ankers like shapes of debris mass are formed due to the splash of the frontal and main debris mass into water. Later, these structures slowly diffuse and change into parabolic shape. These observation and our understanding of tsunami intensity, propagation and the submarine mass movement is useful for hazard mitigation in the tsunami-prone zones.

\section{References}

[1] Charvet I, Suppasri A, Kimura H, Sugawara D and Imamura F (2015) A multivariate generalized linear tsunami fragility model for Kesennuma City based on maximum flow depths, velocities and debris impact, with evaluation of predictive accuracy. Natural Hazards, 97 (doi 10.1007/s11069-0151947-8)

[2] Cui P, Zeng C and Lei Yu (2015) Experimental analysis on the impact force of viscous debris flow. Earth Surf. Process. Landforms, 40, 1644-1655 (doi: 10.1002/esp.3744)

[3] Domnik B, Pudasaini SP, Katzenbach R and Miller SA (2013) Coupling of full two-dimensional and depth-averaged models for granular flows. J. Non-Newtonian Fluid Mech., 201, 56-68

[4] Douglas S (2016) Numerical Modeling of Extreme Hydrodynamic Loading and Pneumatic Long Wave Generation: Application of a Multiphase Fluid Model. PhD Dissertation, University of Ottawa, Canada

[5] Fritz HM (2002) Initial phase of landslide generated impulse waves. PhD Dissertation, Swiss Federal Institute of Technology, ETH Zrich, Switzerland 
Journal of Nepal Mathematical Society (JNMS), Vol. 1, Issue 1 (2018); J. Kafle, B. M. Tuladhar

[6] Fritz HM, Hager WH, Minor H-E (2003) Landslide generated impulse waves .2. hydrodynamic impact craters. Experiments in Fluids, 35, 520-532 (doi: 10.1007s00348-003-0660-7)

[7] Kafle J (2014) Dynamic interaction between a two-phase submarine landslide and a fluid reservoir. MPhil Dissertation, Kathmandu University, School of Science, Dhulikhel, Kavre, Nepal

[8] Kafle J, Pokhrel PR, Khattri KB, Kattel P, Tuladhar BM and Pudasaini SP (2016) Submarine landslide and particle transport in mountain lakes, reservoirs and hydraulic plants. Annals of Glaciology 57(71) (doi: 10.3189/2016AoG71A034)

[9] Kafle J, Kattel P, Mergili M, Fischer J-T, Tuladhar BM and Pudasaini SP (2018) Submarine landslide and tsunami impact on submarine obstacles (submitted)

[10] Kattel P, Khattri KB, Pokhrel PR, Kafle J, Tuladhar BM and Pudasaini SP (2016) Simulating glacial lake outburst floods with a two-phase mass flow model. Annals of Glaciology, 57(71), (doi: 10.3189/2016AoG71A039)

[11] Kattel P, Kafle J, Fischer J-T, Mergili M, Tuladhar BM and Pudasaini SP (2018) Interaction of two-phase debris flow with obstacles (submitted)

[12] Kim G-B (2012) Numerical simulation of three-dimensional tsunami generation by subaerial landslides. Master's Thesis, Texas A\&M University, Texas, USA

[13] Mergili M, Emmer A, Juricov A, Cochachin A, Fischer J-T, Huggel C and Pudasaini SP (2017): How well can we simulate complex hydro-geomorphic process chains? The 2012 multi-lake outburst flood in the Santa Cruz Valley (Cordillera Blanca, Per) Earth Surface Processes and Landforms (Doi: 10.1002/esp.4318)

[14] Miao H, Wang G, Yin K, Kamai T and Li Y (2014) Mechanism of the slow-moving landslides in Jurassic red-strata in the Three Gorges Reservoir, China. Engineering Geology (doi: 10.1016j.enggeo.2013.12.017)

[15] Mohammed F (2010) Physical modeling of tsunamis generated by three-dimensional deformable granular landslides. PhD Dissertation, School of Civil and Environmental Engineering, Georgia Institute of Technology

[16] Mohammed F and Fritz HM (2012) Physical modeling of tsunamis generated by threedimensional deformable granular landslides. Journal of Geophysical Research, 117, 705-718, C11015 (doi:10.1029/2011JC007850)

[17] Pudasaini SP (2012) A general two-phase debris flow model. Journal of Geophysical Research, 117, F03010 (doi:10.1029/2011JF002186)

[18] Pudasaini SP (2014) Dynamics of submarine debris flow and tsunami. Acta Mechanica, 225, 2423-2434 (doi:10.1007/s00707-014-1126-0)

[19] Pudasaini SP and Hutter K (2007) Avalanche Dynamics: Dynamics of Rapid Flows of Dense Granular Avalanches. Springer, Berlin, New York

[20] Tai Y-C, Noelle S, Gray JMNT and Hutter K (2002) Shock-capturing and front-tracking methods for granular avalanches. J. Comput. Phys., 175(1), 269-301

[21] Xiao L, Ward S and Wang J (2015) Tsunami Squares Approach to Landslide-Generated Waves: Application to Gongjiafang Landslide, Three Gorges Reservoir, China. Pure Appl. Geophys., (Doi: $10.1007 / \mathrm{s} 00024-015-10)$

[22] Yalner AC, Pelinovsky E, Okal E and Synolakis CE (2003) Submarine Landslides and Tsunamis. Kluwer Academic Publishers, 9-16 